\title{
More on set-magic graphs
}

\author{
Ullas Thomas \\ Department of Mathematics, St.Berchmans College (Autonomous), Changanacherry, Pin. -686 101, Kottayam, Kerala, India \\ Email: ullasmanickathu@gmail.com,ullasmanickathu@rediffmail.com
}

Copyright (C)2015 Ullas Thomas. This is an open access article distributed under the Creative Commons Attribution License, which permits unrestricted use, distribution, and reproduction in any medium, provided the original work is properly cited.

\begin{abstract}
This paper contains certain properties of set-magic graphs and obtained the set-magic number of certain classes of graphs. All spanning super graphs of a set-magic graph always set-magic and all cycles and Hamiltonian graphs are set-magic. Also set-magic number of any cycle of size $2^{n}$ is always greater than $n$.
\end{abstract}

Keywords: set-magic graph;set-magic labeling; set-magic number; set-valuation.

\section{Introduction}

Graph Labeling is an active area of Graph Theory. Labeling of graphs, is an assignment of finite subset of the set of integers to the vertices or edges, or both, subject to certain conditions. Graph labelings were first introduced in the late 1960 by A. Rosa [5]. It was Acharya [1] who departed from the trend and instead of a finite subset of set of integers, associated an arbitrary non-empty set to the graphs, there by laying the foundations for the set-valuations of graph.

The notion of set-magic graphs was introduced by Sedlacek [4] in 1976 and later some important results of set-magic graphs were in [1] and [2]. This paper contains certain properties of set-magic graphs and obtained the set-magic number of certain classes of graphs. Acharya found certain bound for the set-magic number of any set-magic graph. The class of complete bipartite graphs $K_{2, n}$ for $n>2$ which are set-magic as well as the equality holds for the bound of their set-magic number.

Not all sub graphs and super graphs of set-magic graphs need not be set-magic. But all spanning super graphs of a set-magic graph is always set-magic. All cycles and Hamiltonian graphs are set-magic. Vijayakumar found in

[2] that the set-magic number of any set-magic graph cannot exceed its size. Here the set-magic number of any cycle cannot succeed one less than its size. Also set-magic number of any cycle of size $2^{n}$ is always greater than $n$.

\section{Preliminaries}

In this section we include certain definitions and known results needed for the subsequent development of the study. For a nonempty set $X$, the set of all subsets of $X$ is denoted by $2^{X}$. We always denote a graph under consideration by $G$ and its vertex and edge sets by $V$ and $E$ respectively. By $G^{\prime} \subseteq G$ we mean $G^{\prime}$ is a subgraph of $G$ while $G^{\prime} \subset G$ we mean $G^{\prime}$ is a proper subgraph of $G$. The empty graph of order $n$ is denoted by $N_{n}$. The order and size of a graph $G$ is denoted by $o(G)$ and $s(G)$ respectively. When we say two graphs are different we mean they are non-isomorphic. The basic notations and definitions in graph theory are assumed to be familiar to the reader. 
Definition 2.1 [1] Let $G=(V, E)$ be a given graph and $X$ be a nonempty set. Then a mapping $f: V \rightarrow 2^{X}$, or $f: E \rightarrow 2^{X}$, or $f: V \cup E \rightarrow 2^{X}$ is called a set-assignment or set-valuation of the vertices or edges or both.

Theorem 2.2 [1] Every graph has a set-valuation.

Let $G$ be a graph. For any vertex $v$ of $G$, we denote its neighborhood by $N(v)$ and its degree by $d(v)$. To specify notationally that an edge $e$ of $G$ is incident with $v$, we write $e \sim v$. If an end-vertex of an edge $e$ is of degree 1 , then $e$ is called a pendant edge of $G$.

Definition 2.3 [1] A graph $G=(V, E)$ is said to be set-magic if its edges can be assigned distinct subsets of a set $X$ such that for every vertex at $u$ of $G$, union of the subsets assigned to the edges incident at $u$ is $X$. A graph is said to be set-magic if it admits a set-magic labeling.

If $G$ is a set-magic graph, then the least number that can be the cardinality of a set by which $G$ has a set-magic labeling is called the set-magic number of $G$ and denoted by $\mu(G)$. It is trivial that $\mu\left(K_{2}\right)=0$. A set-magic labeling of $G$ with respect to a set of cardinality $\mu(G)$ be called optimal.

Theorem 2.4 [1] Let $G$ be a graph without isolated vertices. Then $G$ is set-magic if and only if it has at most one pendant edge.

Theorem 2.5 [2] No tree other than $K_{2}$ is set-magic.

Theorem 2.6 [2] If a graph $G=(V, E)$ is set-magic, then $\mu(G) \leq|E|$.

Theorem 2.7 [1] Let $G=(V, E)$ be a set-magic graph, then $\left\lceil\log _{2}|E|\right\rceil \leq \mu(G)$.

\section{More on set-magic graphs}

\section{Remark 1}

There are set-magic graphs with equality as well as strict inequality hold in theorem 2.7.

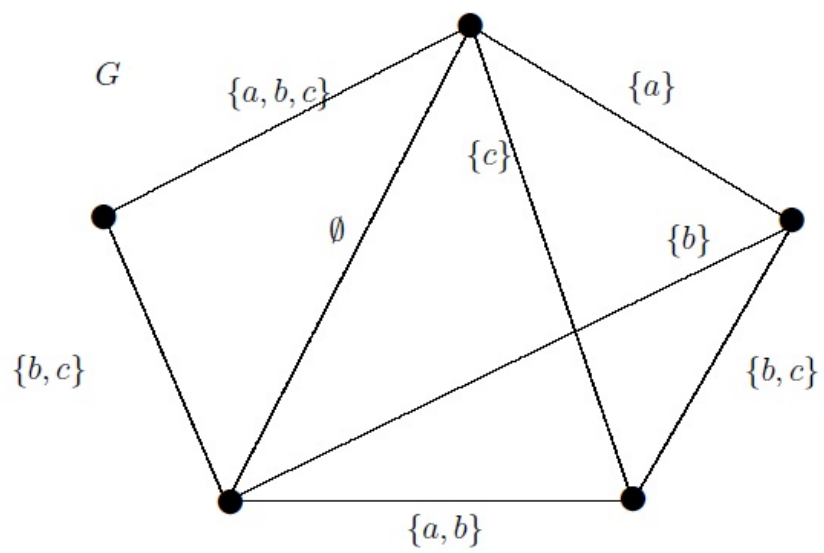

Figure 1: Optimal Set-magic labeling of G. 


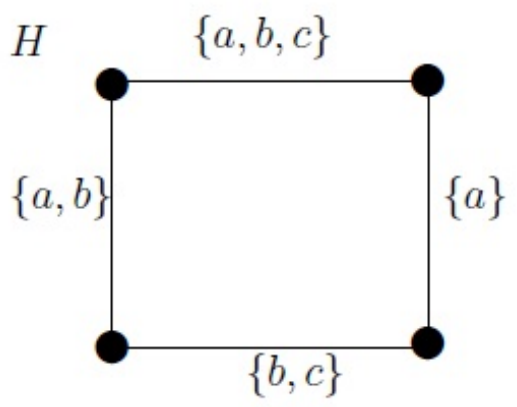

Figure 2: Optimal Set-magic labeling of H.

In the above graph $G, \mu(G)=3=\left\lceil\log _{2}|E|\right\rceil$ and in the graph $H, \mu(H)=3>2=\left\lceil\log _{2}|E|\right\rceil$.

Theorem 3.1 For $n>2$, the complete bipartite graph $K_{2, n}$ is set-magic and $\mu\left(K_{2, n}\right)=\left\lceil\log _{2}|E|\right\rceil$.

ProofLet $V=\left\{u_{1}, u_{2}, v_{1}, \ldots, v_{n}\right\} ; d\left(u_{1}\right)=d\left(u_{2}\right)=n$ and consider a set $X=\left\{x_{1}, \ldots, x_{m}\right\} ; m=\left\lceil\log _{2}|2 n|\right\rceil$. Let $A_{1}, A_{2}, \ldots, A_{2^{m}}$ are distinct subsets of $X$ so that $A_{2^{m-1}+i}=X-A_{i}$ for $i \in\left\{1,2 \ldots, 2^{m-1}\right\}$. Without loss of generality we may assume that $A_{1}=X, A_{2}=\left\{x_{1}\right\}$ and $A_{3}=\left\{x_{2}\right\}$. For $i \in\{1,2, \ldots, n\}$, assign the subsets $A_{i}$ to the edges $\left(u_{1}, v_{i}\right)$ and the subsets $A_{2^{m-1}+i}$ to the edges $\left(u_{2}, v_{i}\right)$ in that order we get a set-valuation $f$ on $K_{2, n}$. Clearly all the edge labels are distinct. Also $f\left(v_{i}\right)=f\left(u_{1}, v_{i}\right) \cup f\left(u_{2}, v_{i}\right)=A_{i} \cup A_{2^{m-1}+i}=A_{i} \cup\left(X-A_{i}\right)=X$ for $1 \leq i \leq n, f\left(u_{1}\right)=f\left(u_{1}, v_{1}\right) \cup f\left(u_{1}, v_{2}\right) \cup \ldots f\left(u_{1}, v_{n}\right)=A_{1} \cup A_{2} \cup \ldots \cup A_{n}=X \cup A_{2} \cup \ldots A_{n}=X$ and $f\left(u_{2}\right)=f\left(u_{2}, v_{1}\right) \cup f\left(u_{2}, v_{2}\right) \cup \ldots f\left(u_{2}, v_{n}\right)=A_{2^{m-1}+1} \cup A_{2^{m-1}+2} \cup \ldots A_{2^{m-1}+n}=\emptyset \cup\left(X-\left\{x_{1}\right\}\right) \cup\left(X-\left\{x_{2}\right\}\right) \cup \ldots=X$ so that all the vertex labels are same as $X$. Hence $f$ is a set-magic labeling with $X$ as the ground set. Thus $K_{2, n}$ is set-magic and by theorem 2.7 we have $m=\left\lceil\log _{2}|E|\right\rceil \leq \mu\left(K_{2, n}\right) \leq|X|=m$.

\section{Remark 2}

$K_{2, n}$ with $n=1$ is not set magic by theorem 2.4.

\section{Remark 3}

In the complete bipartite graph $K_{2, n}$ with $n=2$, then $K_{2,2} \cong C_{4}$. By theorem $2.7,2=\left\lceil\log _{2}|E|\right\rceil \leq \mu\left(C_{4}\right)$. Suppose $\mu\left(C_{4}\right)=2$. Let $f$ be a set-magic labeling of $C_{4}=\left(v_{1}, v_{2}, v_{3}, v_{4}, v_{1}\right)$ with ground set $X=\{a, b\}$. Since all the edge labels are distinct and $|E|=2^{|X|}$ we have $\emptyset$ must be an edge label. Without loss of generality we may assume that $f\left(v_{1}, v_{2}\right)=\emptyset$. But we can assign $X$ to only one edge so that either $f\left(v_{1}\right) \neq X$ or $f\left(v_{2}\right) \neq X$, a contradiction. Thus we get $\mu\left(C_{4}\right)>2=\left\lceil\log _{2}|E|\right\rceil$. Thus the theorem 3.1 on $K_{2, n}$ fails if $n=2$. Also from figure $2, \mu\left(C_{4}\right) \leq 3$ and hence we have $\mu\left(C_{4}\right)=3$.

\section{Remark 4}

Every set-valuation of a graph $G$ is a set-valuation of all of its subgraphs. But every set-magic labeling of a graph need not be a set-magic labeling of all of its subgraphs. The set-magic number of the complete graph $K_{3}$ is 2 . But $K_{2} \cup N_{1}$ is not a set-magic subgraph of $K_{3}$ and $K_{2}$ is a set-magic subgraph of $K_{3}$ with set-magic number 1 .

Now we strongly feel that a relation between set-magic number of a graph and its subgraph is given in the following two conjectures.

\section{Conjecture 1}

Let $G$ be a set-magic graph. Then every set-magic labeling of $G$ is also a set-magic labeling of all of its set-magic subgraphs.

\section{Conjecture 2}

If $G$ is a set-magic graph and $G^{\prime}$ is a subgraph of $G$ which is also set-magic, then $\mu\left(G^{\prime}\right) \leq \mu(G)$.

Theorem 3.2 All disconnected graphs with at least one isolated vertex is not set-magic.

ProofLet $f$ be any set-valuation of the disconnected graph $G$ with ground set $X$. Let $\{v\}$ be an isolated vertex of $G$. Since there is no edge incident with $v$ so that $f$ must not be a set-magic labeling of $G$. Hence $G$ is not a set-magic graph.

\section{Remark 5}

There are disconnected graphs which are set-magic. The graph $C_{3} \cup K_{2}$ is set-magic with ground set $X=\{a, b, c\}$ is given below:

\section{Remark 6}




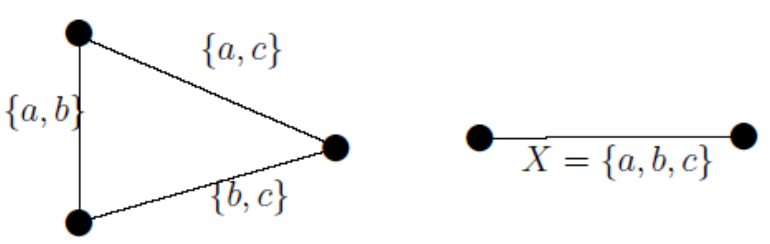

Figure 3: Set-magic Labeling of $C_{3} \cup K_{2}$.

Not all super graphs of a set-magic graph need not be set-magic. By remark $4, K_{3}$ is set-magic. But $K_{3} \cup K_{1}$ - a super graph of $K_{3}$ which is not set-magic by theorem 3.2.

But in the case of spanning super graphs,

Theorem 3.3 If $G$ is a set-magic graph, then all of its spanning super graphs are set-magic.

Proof. Let $f$ be a set-magic labeling of $G=(V, E)$ with ground set $X$. Let $G^{\prime}=\left(V, E^{\prime}\right)$ be a spanning super graph of $G$. Then we have two possibilities.

Case-1 If $\left|E^{\prime}\right| \leq 2^{|X|}$, then we can define a set-valuation $g$ on $G^{\prime}$ as follows: $g(e)=f(e)$ for all $e \in E$. Now we can assign the remaining unused distinct subsets of $X$ to the unassigned edges we get all edge labels are distinct. Also $g(v)=\cup_{e \sim v ; e \in E^{\prime}} g(e)=\left(\cup_{e \sim v ; e \in E} f(e)\right) \cup\left(\cup_{e \sim v ; e \in E^{\prime}-E} g(e)\right)=X ; e \sim v$ denote the set of all edges incident with $v$. Clearly $g$ is a set-magic labeling of $G^{\prime}$ with ground set $X$ and hence $G^{\prime}$ is set-magic.

Case-2 Suppose that $\left|E^{\prime}\right|>2^{|X|}$. Let $Y$ be a nonempty set such that $X \subset Y$ and $|Y|=\left\lceil\log _{2}\left|E^{\prime}\right|\right\rceil$. Define a set-valuation $h$ on $G^{\prime}$ as follows: $h(e)=f(e) \cup(Y-X)$ for all $e \in E$. Now we can assign the remaining unused distinct subsets of $Y$ to the unassigned edges we get all edge labels are distinct. Also $h(v)=\cup_{e \sim v ; e \in E^{\prime}} h(e)=$ $\left(\cup_{e \sim v ; e \in E} f(e) \cup(Y-X)\right) \cup\left(\cup_{e \sim v ; e \in E^{\prime}-E} h(e)\right)=X \cup(Y-X) \cup\left(\cup_{e \sim v ; e \in E^{\prime}-E} h(e)\right)=Y$. Clearly $h$ is a set-magic labeling of $G^{\prime}$ with ground set $Y$ and hence $G^{\prime}$ is set-magic.

Thus in both cases, $G^{\prime}$ - every spanning super graph of a set-magic graph $G$ is set-magic.

Theorem $3.4 \mu\left(C_{2^{n}}\right)>n$ for all $n \geq 2$.

Proof. By theorem 2.7, $n=\left\lceil\log _{2}|E|\right\rceil \leq \mu\left(C_{2^{n}}\right)$. Suppose $\mu\left(C_{2^{n}}\right)=n$. Let $f$ be a set-magic labeling of $C_{2^{n}}=\left(v_{1}, v_{2}, \ldots, v_{2^{n}}, v_{1}\right)$ with ground set $X=\left\{x_{1}, \ldots, x_{n}\right\}$. Since all the edge labels are distinct and $|E|=2^{|X|}$ we have $\emptyset$ must be an edge label. Without loss of generality we may assume that $f\left(v_{1}, v_{2}\right)=\emptyset$. But we can assign $X$ to only one edge so that either $f\left(v_{1}\right) \neq X$ or $f\left(v_{2}\right) \neq X$, a contradiction. Thus we get $\mu\left(C_{2^{n}}\right)>n=\left\lceil\log _{2}|E|\right\rceil$.

Theorem 3.5 All cycles are set-magic and $\mu\left(C_{n}\right) \leq n-1$.

Proof. Let $C_{n}=\left(v_{1}, v_{2}, \ldots, v_{n}, v_{1}\right)$ and $X=\left\{x_{1}, \ldots, x_{n-1}\right\}$. Assign $X-\left\{x_{1}\right\}, X-\left\{x_{2}\right\}, \ldots, X-\left\{x_{n-1}\right\}$ and $X$ to the edges $\left(v_{1}, v_{2}\right),\left(v_{2}, v_{3}\right), \ldots,\left(v_{n-1}, v_{n}\right)$ and $\left(v_{n}, v_{1}\right)$ in that order we get a set-magic labeling of $C_{n}$ and hence $\mu\left(C_{n}\right) \leq n-1$.

\section{Remark 7}

There are cycles with equality as well as inequality holds in theorem 3.5. In Figure 2, we have an optimal set-magic labeling of $C_{4}$ such that $\mu\left(C_{4}\right)=3=4-1$. Now consider the cycle $C_{5}$, 


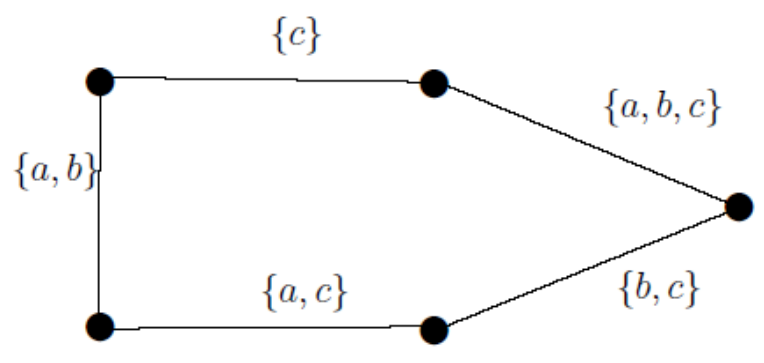

Figure 4: Optimal set-magic labeling of $C_{5}$.

An optimal set-magic labeling of $C_{5}$ with ground set $X=\{a, b, c\}$ is given in Figure 4 and by theorem 2.7 we have, $3=\left\lceil\log _{2}|E|\right\rceil \leq \mu\left(C_{5}\right) \leq 3$. Clearly $\mu\left(C_{5}\right)=3<5-1$, strict ineqality hold in theorem 3.5.

Theorem 3.6 If $G=(V, E)$ be a hamiltonian graph of order $n$, then $G$ is set-magic. If $\mu\left(C_{n}\right)=m$ and $\left\lceil\log _{2}|E|\right\rceil=$ $k ; k \geq m$, then $\mu(G)=k$.

Proof. Since $G$ is a spanning super graph of $C_{n}$ and by theorem 3.5 and theorem 3.3 we have $G$ is set-magic. Let $f$ be an optimal set-magic labeling of $C_{n}$ with ground set $X=\left\{x, \ldots, x_{m}\right\}$.

Case-1 If $k=m$. Then we can extend $f$ as a set-magic labeling of $G$ as follows: Since $|E| \leq 2^{|X|}$, we can assign the remaining subsets of $X$ to the edges of $E-E\left(C_{n}\right)$ in a one to one manner we get all the edge labels of $G$ under $f$ are distinct. Also $f$ is a set-magic labeling of $C_{n}$ so that $f(v)=X$ for all $v \in V\left(C_{n}\right)$ and hence $f(v)=X$ for all $v \in V$. Thus $f$ is a set-magic labeling of $G$. By theorem 2.7 we have, $m=\left\lceil\log _{2}|E|\right\rceil \leq \mu(G) \leq m$.

Case-2 If $k>m$. Then we can find a set-magic labeling $g$ of $G$ with ground set $Y=X \cup\left\{x_{m+1}, \ldots x_{k}\right\}$ as follows: $g(e)=f(e) \cup\left\{x_{m+1}, \ldots x_{k}\right\}$ for all $e \in E\left(C_{n}\right)$. Since $|E| \leq 2^{|Y|}$, we can assign the remaining subsets of $Y$ to the edges of $E-E\left(C_{n}\right)$ in a one to one manner we get all the edge labels of $G$ under $g$ are distinct. Also $g(v)=\cup_{e \sim v ; e \in E} g(e)=\left(\cup_{e \sim v ; e \in E\left(C_{n}\right)} g(e)\right) \cup\left(\cup_{e \sim v ; e \in E-E\left(C_{n}\right)} g(e)\right)=\left(\cup_{e \sim v ; e \in E\left(C_{n}\right)} f(e) \cup\left\{x_{m+1}, \ldots x_{k}\right\}\right) \cup$ $\left(\cup_{e \sim v ; e \in E-E\left(C_{n}\right)} g(e)\right)=\left(X \cup\left\{x_{m+1}, \ldots x_{k}\right\}\right) \cup\left(\cup_{e \sim v ; e \in E-E\left(C_{n}\right)} g(e)\right)=Y \cup\left(\cup_{e \sim v ; e \in E-E\left(C_{n}\right)} g(e)\right)=Y$ for all $v \in V$. Thus $g$ is a set-magic labeling of $G$. By theorem 2.7 we have, $k=\left\lceil\log _{2}|E|\right\rceil \leq \mu(G) \leq k$.

\section{References}

[1] B.D. Acharya, "Set Valuations of a graph and their applications", MRI Lecture Notes in Applied mathematics No.2, Mehta Research Institute, Allahabad, 1983.

[2] B.D. Acharya, S. Arumugam, A. Rosa, Labelings of Discrete Structures and Applications, Narosa Publishing House, New Delhi, 2008.

[3] J.A. Bondy, U.S.R. Murty, Graph Theory with Applications, New York:Macmillan Ltd. Press, 1976.

[4] J. Sedlacek, "Some Properties of Magic Graphs. in Graphs, Hypergraphs and Block Systems" , Proc. Conf. held in Zielona Gora, (1976), pp.247-253.

[5] A. Rosa, "On certain valuations of the vertices of a graph. in: Theory of Graphs, Proc. Intnat. Sympos. Rome, July 1966" Gordon and Breach, (1967), pp.349-355. 